\title{
ON PERFECT MEASURES
}

\author{
BY \\ G. KOUMOULLIS ${ }^{1}$
}

\begin{abstract}
Let $\mu$ be a nonzero positive perfect measure on a $\sigma$-algebra of subsets of a set $X$. It is proved that if $\left\{A_{i}: i \in I\right\}$ is a partition of $X$ with $\mu^{*}\left(A_{i}\right)=0$ for all $i \in I$ and the cardinal of $I$ non-(Ulam-) measurable, then there is $J \subset I$ such that $\bigcup_{i \in J} A_{i}$ is not $\mu$-measurable, generalizing a theorem of Solovay about the Lebesgue measure. This result is used for the study of perfect measures on topological spaces. It is proved that every perfect Borel measure on a metric space is tight if and only if the cardinal of the space is nonmeasurable. The same result is extended to some nonmetric spaces and the relation between perfectness and other smoothness properties of measures on topological spaces is investigated.
\end{abstract}

As many problems of measure theory cannot be solved in general measure spaces, several authors introduced smaller classes of measure spaces ([13], [23] and [24]) so that certain irregularities could be avoided. This paper ${ }^{2}$ is devoted to the class of perfect measures which is wide enough to contain the class of tight (or Radon) measures on topological spaces and have certain stability properties. Special emphasis is laid on perfect measures on topological spaces.

Notation and preliminary results are given in $\S 1$. The main result of $\S 2$ is the first result mentioned in the abstract and it has many applications in the following three sections. In $\$ 3$ we study perfect measures on metric spaces and prove that every perfect measure is tight if and only if the cardinal of the metric space is nonmeasurable.

In $\S 4$ we examine the relation between perfect and $\tau$-additive Borel measures on topological spaces and extend the results of $\S 3$ to some nonmetric spaces. $\$ 5$ is concerned with the comparison of the space $M_{p}$ of perfect measures on a completely regular space with the spaces $M_{\sigma}, M_{\tau}$ and $M_{t}$ of Varadarajan [28]. Examining the spaces for which $M_{p} \subset M_{\tau}$ we obtain an extension of Katetov's theorem on paracompact spaces [7]. Extensions are also given to some results of Sazonov [24], Solovay, and Prikry [21].

1. Preliminaries and notations. Let $(X, \mathcal{Q})$ be a measurable space (i.e. $\mathcal{Q}$ is a $\sigma$-algebra of subsets of $X$ ). A ( $\sigma$-additive finite signed) measure $\mu$ defined on $\mathcal{Q}$ is

Received by the editors July 20, 1979 and, in revised form, April 7, 1980.

AMS (MOS) subject classifications (1970). Primary 28A32, 28A10, 60B05, 28A35; Secondary 54D20, 54D60, 02K 35 .

Key words and phrases. Perfect, $\sigma$-additive, $\tau$-additive, tight measure, measurable cardinal, weakly $\boldsymbol{\theta}$-refinable, weakly metacompact, developable, realcompact space.

${ }^{1}$ The results presented in this paper form a part of the author's doctoral dissertation at Athens University (1978). The dissertation was prepared under the direction of Professor S. Negrepontis whom the author wishes to thank for his encouragement and advice.

${ }^{2}$ Announced in part in Notices Amer. Math. Soc. 26 (1979), A-274.

(C) 1981 American Mathematical Society 0002-9947/81/0000-0166/\$05.25 
perfect if for every $\mathbb{Q}$-measurable function $f: X \rightarrow \mathbf{R}$ there is a Borel set $B$ of $\mathbf{R}$ such that $B \subset f(X)$ and $|\mu|\left(f^{-1}(B)\right)=|\mu|(X)$, where $|\mu|$ is the total variation of $\mu$.

For each $A \subset X$, let $A \cap \mathbb{Q}=\{A \cap B: B \in \mathbb{Q}\}$. If $A \in \mathbb{Q}$ and $\mu$ is a measure on $\mathcal{Q}$, then the measure $\left.\mu\right|_{A}$ is the restriction of $\mu$ to the $\sigma$-algebra $A \cap \mathcal{Q}$ of subsets of $A$ and $\mu_{A}$ is defined on $Q$ by $\mu_{A}(B)=\mu(A \cap B)$. If $(Y, \mathscr{B})$ is another measurable space and $f: X \rightarrow Y$ a measurable function, then for every measure $\mu$ on $\mathbb{Q}$ its image under $f$ is the measure $f_{*}(\mu)$ defined on $\mathscr{B}$ by $f_{*}(\mu)(B)=\mu\left(f^{-1}(B)\right)$. If $A$ is any subset of $X$ and $\mu$ is a measure defined on a $\sigma$-algebra containing $A \cap \mathbb{Q}$ the image of $\mu$ to $X$ is the measure $i_{*}(\mu)$, where $i: A \rightarrow X$ is the inclusion function.

The basic properties of perfect measures are given in the following proposition (cf. Sazonov [24, 1.6]).

1.1. Proposition. Let $(X, \mathbb{Q}, \mu)$ be a positive perfect measure space. Then,

(i) the completion of $\mu$ is perfect;

(ii) for every $A \in \mathbb{Q}, \mu_{A}$ and $\left.\mu\right|_{A}$ are perfect measures;

(iii) for every measurable function $f$ from $X$ into a measurable space $(Y, \mathscr{B})$, the measure $f_{*}(\mu)$ is perfect.

Let $(X, Q, \mu)$ be a positive measure space. If $f$ is a $\mu$-measurable function from $X$ into a measurable space $(Y, \mathscr{B})$ (i.e. $f^{-1}(B)$ is $\mu$-measurable for every $B \in \mathscr{B}$ ), then $f_{*}(\mu)$ means the image of the completion of $\mu$ under $f$. It follows from the preceding proposition ((i) and (iii)) that $f_{*}(\mu)$ is perfect whenever $\mu$ is perfect. By $\mu^{*}$ we denote the Carathéodory outer measure (defined on all subsets of $X$ ). The measure $\mu$ is continuous if $\mu^{*}(\{x\})=0$ for all $x \in X$ and nonatomic if $\mu$ has no atoms (an atom of $\mu$ is an $A \in \mathbb{Q}$ such that $\mu(A)>0$ and $\mu(B)=0$ or $\mu(A)$ for all measurable sets $B \subset A)$. Clearly every nonatomic measure is continuous. If $\mu(X)$ $=1, \mu$ is a probability measure.

1.2. Proposition. If $\mu$ is a nonatomic probability measure, then $\mu$ assumes all values on the unit interval $[0,1]$.

A proof of this proposition is found in $[2$, p. 26] where we observe that the axiom of choice is used only for countable families. Except for Corollary 2.7, where this remark will be used, we shall assume the full axiom of choice.

A (nonzero) measure is 2 -valued if its range is $\{0,1\}$. A cardinal $m$ is (Ulam-) measurable if there is a continuous 2-valued measure defined on all subsets of $m$. Otherwise $m$ is non-(Ulam-) measurable. We refer to [6], [26], [27] for results about measurable cardinals.

The cardinal of a set $A$ will be denoted by $|A|$ and the cardinal of the continuum by $c$.

We now present some basic facts from the theory of measures on topological spaces. Let $X$ be any topological space. A zero set $Z$ of $X$ is a set of the form $Z=f^{-1}(\{0\})$, where $f: X \rightarrow \mathbf{R}$ is continuous. A cozero set of $X$ is a complement of a zero set of $X$. The $\sigma$-algebra $\mathscr{B}(X)$ (resp. $\mathscr{B}_{0}(X)$ ) generated by the zero (resp. closed) sets of $X$ is the family of Baire (resp. Borel) sets of $X$. A Baire (resp. Borel) measure on $X$ is a measure defined on the $\sigma$-algebra of Baire (resp. Borel) sets of $X$. 
If $X$ is a metric space, then any closed set is a zero set and consequently $\mathscr{B}(X)=\mathscr{B}_{0}(X)$. In general $\mathscr{B}(X) \subset \mathscr{B}_{0}(X)$.

A Borel measure $\mu$ on a topological space $X$ is regular if for every $A \in \mathscr{B}_{0}(X)$,

$$
|\mu|(A)=\sup \{|\mu|(F): F \text { closed, } F \subset A\} .
$$

If every closed set of $X$ is a $G_{\delta}$, then every Borel measure on $X$ is regular. Baire measures are necessarily regular (with respect to the zero sets).

The support of a Baire (resp. Borel) measure $\mu$ on $X$ is the intersection of all zero (resp. closed) sets $Z$ with $|\mu|(Z)=|\mu|(X)$.

A net $\left\{Z_{\alpha}\right\}_{\alpha \in A}$ of subsets of $X$ is said to decrease to the set $Z$ and it is denoted by $Z_{\alpha} \downarrow Z$, if $Z_{\alpha} \supset Z_{\beta}$ whenever $\beta>\alpha$, and $\cap_{\alpha \in A} Z_{\alpha}=Z$. An analogous sense is given to the notation $Z_{\alpha} \uparrow Z$. A Baire measure $\mu$ on $X$ is

(i) $\tau$-additive, if whenever a net $\left\{Z_{\alpha}\right\}_{\alpha \in A}$ of zero sets decreases to the empty set, $|\mu|\left(Z_{\alpha}\right) \rightarrow 0$, and

(ii) tight (or Radon), if for every $\varepsilon>0$ there is a compact set $K \subset X$ such that $|\mu|^{*}(K)>|\mu|(X)-\varepsilon$.

$\tau$-additive Borel measures are defined by replacing zero sets by closed sets, while the definition of tight Borel measure is the same, except that $\mu$ is assumed to be regular. Every tight measure is $\tau$-additive.

A (completely) regular space will be assumed to be Hausdorff. The following two results are proved in Kirk [10] (see also Knowles [11]) and Sazonov [24, Theorems 2 and 10] respectively.

1.3. THEOREM. Every $\tau$-additive (resp. tight) Baire measure on a completely regular space has a unique extension to a regular $\tau$-additive (resp. tight) Borel measure.

\subsection{THEOREM. Every tight Baire or Borel measure is perfect.}

In $\S 5$ we will use $M_{\sigma}(X)$ to denote the linear space of all ( $\sigma$-additive) Baire measures on $X$ and $M_{p}(X), M_{\tau}(X)$ and $M_{t}(X)$ to denote the subspaces of perfect, $\tau$-additive and tight Baire measures on $X$. The subsets of positive measures in these spaces are $M_{\sigma}^{+}(X), M_{p}^{+}(X), M_{\tau}^{+}(X)$ and $M_{t}^{+}(X)$. We have

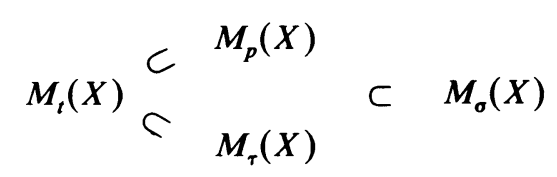

In $\S \S 2-4$ we will consider only positive measures. One reason is that perfect, $\tau$-additive, tight and regular measures are defined using the total variation measure.

2. Perfect measures. We use $\lambda$ to denote the Lebesgue measure on Borel sets of $[0,1]$.

2.1. Theorem. If $(X, \mathcal{Q}, \mu)$ is a probability nonatomic measure space, then there is a measurable function from $X$ into $[0,1]$ such that $f_{*}(\mu)=\lambda$. If moreover $\mu$ is perfect, $f$ can be chosen onto.

We will use the following. 
2.2. LemMA. Let $C$ be a compact subset of $[0,1]$ and $\mu$ a probability continuous measure on $C$. Then there is an increasing continuous function $h$ from $C$ onto $[0,1]$ such that $h_{*}(\mu)=\lambda$.

Proof. Let $\bar{\mu}$ be the image of $\mu$ to $[0,1]$. We define $f:[0,1] \rightarrow[0,1]$ by $f(x)=\bar{\mu}([0, x])$. Then $f$ is continuous (since $\bar{\mu}$ is continuous) and increasing. Moreover, $f(0)=0$ and $f(1)=1$, so $f$ is onto. Let $h$ be the restriction of $f$ to $C$. If $x \in[0,1] \backslash C$ then

$$
\min \{|x-y|: y \in C\}=\left|x-x_{0}\right|
$$

for some $x_{0} \in C$ and it is immediate that $f(x)=f\left(x_{0}\right)=h\left(x_{0}\right)$. Therefore, $h$ is onto. It is easy to see that $h_{*}(\mu)([0, \alpha])=\alpha$ for all $\alpha \in[0,1]$, hence $h_{*}(\mu)=\lambda$.

Proof of Theorem 2.1. Since $\mu$ is nonatomic, for every $n \in \mathbf{N}$ we can find a finite partition $\bigodot_{n}$ of $X$ so that $\bigodot_{n} \subset \mathbb{Q}$ and $\mu(A) \leqslant 1 / n$ for all $A \in \mathcal{C}_{n}$ (Proposition 1.2). The family $e=\cup_{n} e_{n}$ is countable, so let $巳=\left\{A_{1}, A_{2}, \ldots\right\}$. We define $g$ from $X$ into the Cantor set $C=\{0,1\}^{\mathrm{N}}$ by

$$
g(x)(k)=\left\{\begin{array}{ll}
1, & \text { if } x \in A_{k}, \\
0, & \text { if } x \notin A_{k},
\end{array} \quad k=1,2, \ldots\right.
$$

Then, $g^{-1}(\mathscr{B}(C))=\sigma(\mathcal{C})$ (the $\sigma$-algebra generated by $\mathcal{C}$ ) and it is easy to see that the measure $\nu=g_{*}(\mu)$ is continuous on $C$. By Lemma 2.2, there is $h: C \rightarrow[0,1]$ continuous such that $h_{*}(\nu)=\lambda$. Then the function $f=h \circ g$ is as desired.

If $\mu$ is perfect, then $\nu$ is supported by the union of a pairwise disjoint (possibly finite) sequence $\left\{C_{n}\right\}$ of compact sets contained in $g(X)$. By Lemma 2.2, there are continuous functions $h_{n}$ from $C_{n}$ onto $[0,1]$ such that $\left(h_{n}\right)_{*}\left(\left.\nu\right|_{C_{n}}\right)=\nu\left(C_{n}\right) \cdot \lambda$. We define $h: C \rightarrow[0,1]$ by

$$
h(x)=\left\{\begin{array}{l}
h_{n}(x), \quad \text { if } x \in C_{n}, \\
0, \quad \text { if } x \notin \cup_{n} C_{n} .
\end{array}\right.
$$

Then the function $f=h \circ g$ is onto and $f_{*}(\mu)=\lambda$.

Remark. Let $(X, \mathcal{Q}, \mu)$ be a measure space. A sub- $\sigma$-algebra $\mathscr{B}$ of $\mathcal{Q}$ is called

(i) $\mu$-equivalent to $\mathscr{Q}$, if for every $A \in \mathbb{Q}$ there is $B \in \mathscr{B}$ such that $\mu(A \triangle B)=$ 0 , and

(ii) separable, if it is generated by countably many sets.

The measure $\mu$ is called separable, if there is a separable sub- $\sigma$-algebra $\mu$-equivalent to $Q$.

The following theorem is due to Zink [29]:

"If $\mu$ is a separable probability measure, then there is a measurable function $f$ : $X \rightarrow[0,1]$ such that $f^{-1}(\Re([0,1]))$ is $\mu$-equivalent to $Q$ and $f_{*}(\mu)=\lambda$."

This result is an extension of a classical theorem of Halmos and von Neumann (or Carathéodory) (see [5, p. 173]). Zink's proof is based on this theorem. A simpler direct proof follows from the proof of Theorem 2.1: The proof of 2.1 remains unchanged if $\mathcal{C}$ is enlarged by countably many elements of $\mathscr{Q}$ and especially, since $\mu$ is separable, by those elements generating a sub- $\sigma$-algebra $\mathscr{B} \mu$-equivalent to $\mathscr{Q}$. Then, $f^{-1}(\mathscr{B}([0,1]))$ is $\mu$-equivalent to $\mathscr{C}$, since $g^{-1}(\mathscr{B}(C))=\sigma(\mathcal{C}) \supset \mathscr{B}$ and $h^{-1}(\mathscr{B}([0,1]))$ is $g_{*}(\mu)$-equivalent to $\mathscr{B}(C)$ because $h$ of Lemma 2.2 is increasing and $g_{*}(\mu)$ continuous. 
The conclusion of Theorem 2.1 characterizes nonatomic measures. Also, $f^{-1}(\mathscr{B}([0,1]))$ is a separable sub- $\sigma$-algebra on which $\mu$ remains nonatomic. So we have the following.

2.3. Corollary. Let $(X, Q, \mu)$ be a measure space. The following are equivalent:

(i) $\mu$ is a nonatomic measure;

(ii) there is a measurable function $f: X \rightarrow[0,1]$ such that $f_{*}(\mu)=\mu(X) \cdot \lambda$;

(iii) there is a separable sub- $\sigma$-algebra $\mathscr{B}$ of $\mathbb{Q}$ such that the restriction of $\mu$ to $\mathscr{B}$ is a nonatomic measure.

2.4. Corollary. Let $(X, Q, \mu)$ be a perfect nonatomic measure space. Then,

(i) every $A \in \mathbb{Q}$ with $\mu(A)>0$ has a partition $\left\{A_{i}: i \in I\right\}$ of nonempty measurable sets such that $\mu\left(A_{i}\right)=0$ for all $i \in I$ and $|I|=c$;

(ii) every $A \in \mathbb{Q}$ with $\mu(A)>0$ contains a measurable set $B$ such that $\mu(B)=0$ and $|B| \geqslant c$.

Proof. We can assume that $A=X$ (Proposition 1.1) and $\mu(X)=1$. By Theorem 2.1 there is a measurable function $f$ from $X$ onto $[0,1]$ such that $f_{*}(\mu)=\lambda$. Then $\left\{f^{-1}(\{x\}): x \in[0,1]\right\}$ is a partition of $X$ as in (i). If $C \subset[0,1]$ is the Cantor set, let $B=f^{-1}(C)$. Then $|B| \geqslant|C|=c$ and $\mu(B)=\lambda(C)=0$ which proves (ii).

Part (i) of the above corollary with $A_{i}$ not necessarily nonempty is of course valid for every nonatomic measure. Part (ii) has been proved by Marczewski [13, 7(iii)] for the smaller class of compact measures.

2.5. TheOREM. Let $(X, Q, \mu)$ be a perfect measure space, $\mu \neq 0$, and $\left\{A_{i}: i \in I\right\} a$ partition of $X$ such that $\mu^{*}\left(A_{i}\right)=0$ for all $i \in I$ and $|I|$ nonmeasurable cardinal. Then there is $J \subset I$ such that $\cup_{i \in J} A_{i}$ is not $\mu$-measurable.

Proof. Define $f: X \rightarrow I$ such that $\left.f\right|_{A_{i}}=i$ (if $A_{i} \neq \varnothing$ ). Consider on $I$ the $\sigma$-algebra of all subsets and suppose that the result is not valid. Then $f$ is $\mu$-measurable and the measure $\nu=f_{*}(\mu)$ defined on all subsets of $I$ is perfect (Proposition 1.1) nonzero and continuous. Moreover, $\nu$ is nonatomic. Indeed, if $A$ were an atom of $\nu$, then $(1 / \nu(A)) \cdot \nu_{A}$ would be a continuous 2-valued measure defined on all subsets of $I$, contrary to the assumption that $|I|$ is nonmeasurable.

By Corollary 2.3 there is a function $g: I \rightarrow[0,1]$ such that $g_{*}(\nu)=\nu(X) \cdot \lambda$. Since $\nu$ is defined on all subsets of $I, \lambda$ can be extended to a measure $\tilde{\lambda}$ on all subsets of $[0,1]$ which is also perfect (Proposition 1.1(iii)). As there are nonLebesgue-measurable subsets of $[0,1], \tilde{\lambda}$ is not the completion of the Lebesgue measure, so we can find $A \subset[0,1]$ such that

$$
\tilde{\lambda}(A)>\sup \{\lambda(B): B \text { Borel, } B \subset A\} .
$$

If we consider the inclusion function $h: A \rightarrow \mathbf{R}$, it follows . mmediately that $\tilde{\lambda} \mid A$ is not perfect, contrary to Proposition 1.1(ii).

2.6. Corollary. A cardinal $m$ is nonmeasurable if and only if every perfect continuous measure defined on all subsets of $m$ is identically zero.

Proof. If $m$ is measurable then the 2-valued continuous measure defined on all subsets of $m$ (by the definition of measurable cardinals) is nonzero and perfect. The converse follows from Theorem 2.5 . 
2.7. Corollary. Using the axiom of choice only for countable families, the following are equivalent:

(i) There is a non-Lebesgue-measurable subset of [0, 1];

(ii) every perfect continuous measure defined on all subsets of any set of nonmeasurable cardinal is identically zero;

(iii) every perfect continuous measure defined on all subsets of any set of cardinal at most $c$ is identically zero.

Proof. (i) $\Rightarrow$ (ii) follows from Corollary 2.6. The existence of a non-Lebesguemeasurable set was used in the proof of Theorem 2.5, and the axiom of choice for countable families is needed to apply Proposition 1.2 in the proof of Theorem 2.1.

(ii) $\Rightarrow$ (iii) is obvious.

(iii) $\Rightarrow$ (i). Suppose that every subset of $[0,1]$ is Lebesgue measurable. Then the completion $\tilde{\lambda}$ of the Lebesgue measure $\lambda$ is a nonzero, perfect (Proposition 1.1(i)) continuous measure defined on all subsets of $[0,1]$, which contradicts (iii).

REMARKS. An immediate consequence of Theorem 2.5 is that if $(X, \mathcal{Q}, \mu)$ is a nonzero perfect measure space and either $\mu$ is continuous and $|X|$ nonmeasurable or $\mu$ is nonatomic, then there is a non- $\mu$-measurable set. Indeed, in any case we can find a partition of $X$ as in the statement of Theorem 2.5 (cf. also Corollary 2.4(i)).

Theorem 2.5 generalizes the following theorem due to Solovay about the Lebesgue measure $\lambda$ on $[0,1]$.

2.8. TheOREM. If $\left\{A_{i}: i \in I\right\}$ is a partition of $[0,1]$ such that $\lambda^{*}\left(A_{i}\right)=0$ for all $i \in I$, then there is $J \subset I$ such that $\cup_{i \in J} A_{i}$ is not Lebesgue measurable.

From Prikry [21] we were informed that Solovay's proof employed some fairly sophisticated facts about real-valued measurability and that Bukovsky gave another metamathematical proof of this theorem (using Boolean-valued models). A relatively simple proof using Fubini's theorem is given by Prikry in [21]. It should be observed that essentially the same Prikry's proof can be used to show that the above theorem is valid for some nonperfect measures (measures on separable and separated $\sigma$-algebras; a $\sigma$-algebra $\mathcal{Q}$ of $X$ is separated if for every $x, y \in X, x \neq y$, there is $A \in Q$ such that $x \in A$ and $y \notin A$ ).

A cardinal $m$ is of measure zero if every continuous measure defined on all subsets of $m$ is identically zero. Clearly every cardinal of measure zero is nonmeasurable. Under the continuum hypothesis $\left(c=\aleph_{1}\right)$, the converse is also true (Ulam [27]). Therefore Theorems 2.5 and 2.8 become trivial if we assume that $|I|$ is of measure zero or use the continuum hypothesis. But it should be noted that, without any set theoretical assumption, it has not yet been proved that $c$ is of measure zero. Also, while the cardinal of the continuum is encountered frequently, measurable cardinals do not appear in most mathematical work.

Sazonov in [24, Lemma 4] proves that there does not exist a nonzero perfect continuous measure defined on all subsets of $[0,1]$. It is this result that led us to characterize cardinals with this property in Corollary 2.6.

It is known [25] that (i) of Corollary 2.7 cannot be proved using the axiom of choice only for countable families. Thus, the same is true for (ii) and (iii). 
3. Perfect measures on metric spaces. In this section we examine perfect measures defined on the Borel (= Baire) sets of a metric space. For our main Theorem 3.2, which is an application of Theorem 2.5, the following result of Sazonov [24] is also needed.

3.1. Proposition. Every perfect Borel measure on a separable metric space is tight.

3.2. TheOREM. Let $X$ be a metric space and $\mu$ a Borel measure on $X$. Then, $\mu$ is a tight measure if and only if $\mu$ is perfect and there is a subset $R$ of $X$ of nonmeasurable cardinal such that $\mu^{*}(R)=\mu(X)$.

Proof. If $\mu$ is tight, then $\mu$ is perfect (Theorem 1.4) and $\mu(X)=\mu(C)$ for some $\sigma$-compact set $C$ which has cardinal at most $c$.

Conversely, suppose that $\mu$ is perfect and $\mu^{*}(R)=\mu(X)$ for some set $R$ of nonmeasurable cardinal. The support $S$ of $\mu$ is separable since it satisfies the countable chain condition and, by Proposition 3.1, it suffices to show that $\mu(S)=$ $\mu(X)$.

Let $\mathscr{Q}=\{G \subset X$ : $G$ open, $\mu(G)=0, G \cap R \neq \varnothing\}$. By Stone's theorem there is a $\sigma$-discrete refinement $\mathscr{V}=\cup{ }_{n=1}^{\infty} \mathcal{V}_{n}$, each $\mathcal{V}_{n}$ discrete, $\cup \mathcal{V}=\cup \mathscr{U}$. Since $\mu^{*}(R)=\mu(X)$, we have $\mu(\cup \mathscr{Q})=\mu(X \backslash S)$ and it suffices to show that $\mu(\cup \mathscr{Q})$ $=0$. If $\mu(\cup \mathscr{U})>0$, then for some $n \in N, \mu\left(\cup \mathcal{V}_{n}\right)>0$. Fix such an $n$.

The measure $\left.\mu\right|_{\cup \mathscr{V}_{n}}$ is perfect, nonzero, each member of $\mathscr{V}_{n}$ is open of measure zero and $\left|\mathcal{V}_{n}\right|$ is nonmeasurable (since $|R|$ is nonmeasurable). Hence, by Theorem 2.5 , there is $\mathscr{W} \subset \mathscr{V}_{n}$ such that $\cup \mathcal{W}$ is not $\mu$-measurable. This is a contradiction since $\cup \mathscr{W}$ is open.

Note. The use of Stone's theorem in the above proof can be avoided if we appeal to the following more elementary but less known result of Montgomery (cf. [16, Lemma 2]): Let $\left\{G_{\alpha}: \alpha \in A\right\}$ be a family of open subsets of a metric space, where $A$ is a well ordered set, and

$$
H_{\alpha}=G_{\alpha} \backslash \bigcup_{\beta<\alpha} G_{\beta}, \quad \alpha \in A .
$$

Then for every $E \subset A$, the set $\cup_{\alpha \in E} H_{\alpha}$ is an $F_{\sigma}$.

3.3. Corollary. A metric space $X$ is of nonmeasurable cardinal if and only if every perfect Borel measure on $X$ is tight.

Proof. If the cardinal of $X$ is measurable, then the 2-valued continuous measure $\mu$ defined on all subsets of $X$ is perfect and every subset of $X$ of nonmeasurable cardinal has $\mu$-measure zero. Then the restriction of $\mu$ to the Borel sets is perfect but not tight, since compact sets have measure zero. The converse follows from Theorem 3.2.

3.4. Corollary. Let $(S, \mathcal{Q}, \mu)$ be a perfect measure space, $X$ a metric space and $f$ : $S \rightarrow X$ a $\mu$-measurable function such that $|f(S)|$ is nonmeasurable. Then there is a $\sigma$-compact subset $Y$ of $X$ such that $\mu\left(f^{-1}(Y)\right)=\mu(S)$.

Proof. By Proposition 1.1, $\nu=f_{*}(\mu)$ is perfect and $\nu^{*}(f(S))=\nu(X)$. It follows from Theorem 3.2 that $\nu$ is tight. So there is a $\sigma$-compact subset $Y$ of $X$ such that $\nu(X)=\nu(Y)$, that is, $\mu(S)=\mu\left(f^{-1}(Y)\right)$. 
3.5. Corollary. Let $X$ be a metric space of nonmeasurable cardinal.

(i) $A$ measure $\mu$ on a $\sigma$-algebra $\mathbb{Q}$ containing the Borel sets of $X$ is perfect if and only if $\mu(A)=\sup \{\mu(K): K$ compact, $K \subset A\}$ for all $A \in \mathbb{Q}$.

(ii) If $\mu$ is a perfect Borel measure on $X$, then its completion $\tilde{\mu}$ has no proper extension to a perfect measure.

Proof. (i) Suppose that $\mu$ is perfect and $A \in \mathcal{Q}$. By Proposition $1.1,\left.\mu\right|_{A}$ is a perfect measure on Borel sets of $A$, hence a tight measure (Corollary 3.3). Therefore, $\mu(A)=\sup \{\mu(K): K$ compact, $K \subset A\}$.

Conversely, if $\mu$ satisfies this relation for all $A \in \mathbb{Q}$, then its restriction $\nu$ to the Borel sets is tight, hence perfect. It is evident that $\mu$ is the restriction to $\mathbb{A}$ of the completion of $\nu$, hence perfect (Proposition 1.1).

(ii) By Proposition 1.1, $\tilde{\mu}$ is perfect. The conclusion follows from (i).

REMARKs. Corollary 3.3 contains the result of Sazonov [24] that in a metric space in which every family of disjoint nonempty open sets has cardinal at most $c$ every perfect Borel measure is tight.

Corollary 3.4 is easily proved equivalent to the case of Theorem 2.5 when $X$ is discrete. It generalizes a strong form (with $\sigma$-compact replacing separable) of the following theorem due to Solovay (see Prikry [21]):

3.6. Theorem. Let $X$ be a metric space and $f:[0,1] \rightarrow X$ a Lebesgue-measurable function. Then there is a separable subset $Y$ of $X$ such that $f^{-1}(Y)$ has Lebesgue measure one.

Part (i) of Corollary 3.5 has been proved by Sazonov [24, Theorem 12] for separable metric spaces, and part (ii) by Ryll-Nardzewski [23, p. 130] for the Lebesgue measure.

4. Perfect Borel measures. The results of the preceding section will be extended to some nonmetric spaces (Theorem 4.13). First, we examine the relation between perfect and $\tau$-additive Borel measures on some more general topological spaces (defined below). We prove (Theorem 4.3) that, if closed discrete subsets in these spaces have nonmeasurable cardinal, then every perfect regular Borel measure is $\tau$-additive. The proof is based on arguments used by Haydon in [4, Proposition 3.2] and Gardner in [3, Theorem 3.9] and uses Theorem 2.5. The same method can be used to show that Theorem 3.9 of Gardner [3] is true if we assume that closed discrete subsets (not all discrete subsets) of the space have cardinal of measure zero. Using the continuum hypothesis, Gardner's theorem with this statement implies Theorem 4.3, but without any set theoretical assumption this is not possible even if closed discrete subsets have cardinal at most $c$ (cf. comments following Theorem 2.8 about cardinals of measure zero).

4.1. Definitions. (i) A topological space $X$ is weakly metacompact if for every open cover $\mathscr{Q}$ of $X$ there is an open cover $\mathcal{V}=\cup_{n=1}^{\infty} \mathcal{V}_{n}$ such that $\mathcal{V}$ refines $\mathscr{U}$ and each $\mathcal{V}_{n}$ is point finite.

(ii) A topological space $X$ is weakly $\theta$-refinable if for every open cover $\mathcal{Q}$ of $X$ there is an open cover $\mathscr{V}=\cup_{n=1}^{\infty} \mathcal{V}_{n}$ which refines $\mathcal{U}$ and which has the property 
that if $x \in X$, then there is an $n$ such that $x$ belongs to at least one but at most finite members of $\mathcal{V}_{n}$.

Clearly, every weakly metacompact space is weakly $\boldsymbol{\theta}$-refinable.

The Sorgenfrey line $S$ is the real line with the topology which has for a base the family of all half-open intervals $[x, y), x<y$. Finite and countable products of $S$ are weakly $\theta$-refinable spaces and their closed sets are $G_{\delta}$ 's (see [12] where weakly $\theta$-refinable spaces were introduced and the references given there). In the sequel we shall use only the Sorgenfrey plane $S^{2}$ as a counterexample.

4.2. LeMmA. Let $\mu$ be a Borel measure on a topological space $X$.

(i) If $\mu$ is regular $\tau$-additive and $C$ is the support of $\mu$, then $\mu(C)=\mu(X)$.

(ii) If $\mu$ is not $\tau$-additive, then there is a closed subset $F$ of $X$ such that $\mu_{F}$ is a nonzero Borel measure with empty support (cf. [17, Theorem 2.1]).

Proof. (i) Let

$$
\mathscr{F}=\{F \subset X: F \text { closed, } \mu(F)=\mu(X)\} .
$$

Then $C=\cap \mathscr{F}$ is closed. Labelling $\mathscr{F}$ as $\left(F_{\alpha}\right)_{\alpha \in \mathscr{Q}}$ we make $\mathcal{Q}$ into a directed set by saying that $\alpha>\beta$ if and only if $F_{\alpha} \subset F_{\beta}$. That this partial order makes $Q$ a directed set is clear since if $F_{\alpha}, F_{\beta} \in \mathcal{F}$ then $F_{\alpha} \cap F_{\beta} \in \mathscr{F}$. Suppose that $\mu(C)<$ $\mu(X)-\delta$ for some $\delta>0$. By the regularity of $\mu$, there is an open subset $G$ of $X$ such that $C \subset G$ and $\mu(G) \leqslant \mu(X)-\delta$. Then

$$
\mu\left(F_{\alpha} \backslash G\right)=\mu\left(F_{\alpha}\right)-\mu\left(F_{\alpha} \cap G\right) \geqslant \mu(X)-(\mu(X)-\delta)=\delta>0
$$

for all $\alpha \in Q$. So $F_{\alpha} \backslash G \downarrow \varnothing$, every $F_{\alpha} \backslash G$ is closed and $\mu\left(F \backslash G_{\alpha}\right) \geqslant \delta$, which contradicts the $\tau$-additivity of $\mu$. Therefore, $\mu(C)=\mu(X)$.

(ii) Since $\mu$ is not $\tau$-additive there is a net $\left(G_{\alpha}\right)_{\alpha \in \mathscr{Q}}$ of open subsets of $X$ such that $G_{\alpha} \uparrow X$ and $\mu\left(G_{\alpha}\right) \rightarrow a<\mu(X)$. Let $\left(\alpha_{n}\right)$ be an increasing sequence in $\mathcal{Q}$ such that $\mu\left(G_{\alpha_{n}}\right) \rightarrow a$. We set $F=X \backslash \cup{ }_{n=1}^{\infty} G_{\alpha_{n}}$. Then $F$ is closed and $\mu(F)=\mu(X)-a>$ 0 , so $\nu=\mu_{F}$ is a nonzero Borel measure. For every $\alpha \in \mathbb{Q}$

$$
\nu\left(G_{\alpha}\right)=\mu\left(G_{\alpha} \backslash \bigcup_{n=1}^{\infty} G_{\alpha_{n}}\right)=0
$$

and $X=\cup_{\alpha \in \mathscr{Q}} G_{\alpha}$, so $\nu$ has empty support.

4.3. TheOREM. Let $X$ be a weakly $\theta$-refinable $T_{1}$-space and $\mu$ a perfect regular Borel measure on $X$. We suppose that there is a subset $R$ of $X$ such that every closed discrete subset of $R$ has nonmeasurable cardinal and $\mu^{*}(R)=\mu(X)$. Then $\mu$ is $\tau$-additive.

Proof. We assume that $\mu$ is not $\tau$-additive. By Lemma 4.2(ii) there is a closed $F \subset X$ such $\nu=\mu_{F}$ is a nonzero Borel measure with empty support. Also $\nu$ is perfect (Proposition 1.1) and regular. For every $x \in X$ let $V_{x}$ be an open set with $x \in V_{x}$ and $\nu\left(V_{x}\right)=0$. Since $X$ is weakly $\theta$-refinable there is an open cover $\mathcal{V}=\cup_{n=1}^{\infty} \mathcal{V}_{n}$ of $X$ which refines $\left(V_{x}\right)_{x \in X}$ and which has the property that if $x \in X$, then there is an $n$ such that $x$ belongs to at least one but at most finite members of $\mathcal{V}_{n}$. 
For each $m, n \geqslant 1$ let $X_{n}^{m}$ be the set of all points of $X$ that belong to at least one but at most $m$ members of $\mathfrak{V}_{n}$. Then $X=\cup_{n=1}^{\infty} \cup_{m=1}^{\infty} X_{n}^{m}$. The sets $X_{n}^{m}$ are Borel sets. Indeed, we have $X_{n}^{m} \subset \cup \mathfrak{V}_{n}$. If $x \in \cup \mathfrak{V}_{n} \backslash X_{n}^{m}$, then there are $m+1$ different members $V_{i}, i=1, \ldots, m+1$, of $\mathcal{V}_{n}$ such that $x \in \cap_{i=1}^{m+1} V_{i}$. Then $\cap_{i=1}^{m+1} V_{i} \subset \cup \mathfrak{V}_{n} \backslash X_{n}^{m}$. Therefore $X_{n}^{m}$ is closed in $\cup \mathfrak{V}_{n}$, hence Borel in $X$. Let $X_{n}=\cup_{m=1}^{\infty} X_{n}^{m}$. We fix an $n$ such that $\nu\left(X_{n}\right)>0$. Since $X_{n}^{m} \uparrow X_{n}$, there is an $m>1$ such that $\nu\left(X_{n}^{m} \backslash X_{n}^{m-1}\right)>0$ (we set $X_{n}^{0}=\varnothing$ ). By the regularity of $\nu$, there is a closed set $Y$ such that $Y \subset X_{n}^{m} \backslash X_{n}^{m-1}$ and $\nu(Y)>0$.

Every point of $Y$ belongs to exactly $m$ different members of $\mathcal{V}_{n}$. Let $\mathcal{C}$ be the open cover of $Y$ of all $Y \cap \cap{ }_{i=1}^{m} V_{i}$, where $V_{i} \in \mathscr{V}_{n}, i=1, \ldots, m$, are distinct. Then $\mathcal{C}$ is a partition of $Y$. Let $\tilde{\complement}$ be the family of all $G \in \mathcal{C}$ with $G \cap R \neq \varnothing$. For each $G \in \tilde{\mathcal{C}}$, choose $x_{G} \in G \cap R$ and let $S=\left\{x_{G}: G \in \tilde{\mathcal{C}}\right\}$. We have that $S$ is closed (using the $T_{1}$-property) and discrete, so $|\tilde{\mathcal{E}}|=|S|$ is nonmeasurable. The union of every subfamily of $\tilde{\mathcal{C}}$ is open in $Y$, hence Borel in $X$. Let $W=\cup \tilde{e}$. The measure $\left.\nu\right|_{W}$, defined on the Borel sets of $W$, is perfect (Proposition 1.1), $\left.\nu\right|_{W}(W)$ $=\nu(\cup \tilde{e})=\nu(\cup \mathcal{C})=\nu(Y)>0\left(\right.$ since $\left.\nu^{*}(R)=\nu(X)\right)$, and $\left.\nu\right|_{W}(G)=0$ for all $G \in \tilde{\mathcal{C}}$, contrary to Theorem 2.5 . Therefore $\mu$ is $\tau$-additive.

4.4. Corollary. Let $X$ be a weakly $\theta$-refinable $T_{1}$-space. Then, every perfect regular Borel measure on $X$ is $\tau$-additive if and only if every closed discrete subset of $X$ has nonmeasurable cardinal.

Proof. Suppose that there is a closed discrete $F \subset X$ with measurable cardinal and let $\mu$ be a 2-valued continuous measure on all subsets of $F$. Then its image $\bar{\mu}$ to $X$ is a perfect regular Borel measure with empty support; hence it is not $\tau$-additive (Lemma 4.2(i)).

The converse follows from Theorem 4.3.

If we restrict the cardinality of every discrete subset of $R$, Theorem 4.3 is true when $\mu$ is not necessarily regular. In this case we do not need the $T_{1}$-property.

4.5. TheOREM. Let $X$ be a weakly $\theta$-refinable space and $\mu$ a perfect Borel measure on $X$. We suppose that there is a subset $R$ of $X$ such that discrete subsets of $R$ have nonmeasurable cardinal and $\mu^{*}(R)=\mu(X)$. Then $\mu$ is $\tau$-additive.

The proof is the same except that $Y$ is replaced by $X_{n}^{m} \backslash X_{n}^{m-1}$.

4.6. COROLlary. Let $X$ be a regular hereditary weakly $\theta$-refinable space such that every discrete subset of $X$ has nonmeasurable cardinal. Then every perfect Borel measure on $X$ is regular.

Proof. Let $\mu$ be a perfect Borel measure on $X$. If $\left\{G_{\alpha}\right\}_{\alpha \in \mathscr{Q}}$ is a net of open subsets of $X$ such that $G_{\alpha} \uparrow G$, then by Theorem $4.5, \mu\left(G_{\alpha}\right) \rightarrow \mu(G)$ since $G$ is weakly $\theta$-refinable. By the regularity of $X$, it is easy to see that $\mu$ is regular (cf. [3, Theorem 5.4]).

For weakly metacompact spaces Theorem 4.3 takes the following form. 
4.7. TheOREM. Let $X$ be a weakly metacompact $T_{1}$-space and $\mu$ a perfect regular Borel measure on $X$. Then the following are equivalent:

(i) $\mu$ is $\tau$-additive;

(ii) there is a closed Lindelöf subset $Y$ of $X$ such that $\mu(Y)=\mu(X)$;

(iii) there is a subset $R$ of $X$ such that every closed discrete subset of $R$ has nonmeasurable cardinal and $\mu^{*}(R)=\mu(X)$.

Proof. In analogy to a result of Moran [19, Theorem 5.1] on Baire measures, we can prove that the support of a $\tau$-additive Borel measure on a weakly metacompact space is Lindelöf. Therefore the equivalence (i) $\Leftrightarrow$ (ii) (for any regular $\mu$ ) follows from Lemma 4.2(i).

(ii) $\Rightarrow$ (iii) is trivial and (iii) $\Rightarrow$ (i) follows from Theorem 4.3.

4.8. EXAMPLE. The Sorgenfrey plane $S^{2}$ is weakly $\theta$-refinable and closed sets in $S^{2}$ are $G_{\delta}$ 's. Therefore every Borel measure is regular and, by Theorem 4.3, every perfect Borel measure is $\tau$-additive.

Let $r_{n}, n=1,2, \ldots$, be an enumeration of all rational numbers and define $\varphi_{n}$ : $\mathbf{R} \rightarrow S^{2}$ by $\varphi_{n}(x)=\left(x, x+r_{n}\right)$ for $n=1,2, \ldots$ Then $\varphi_{n}$ is a Borel isomorphism of $\mathbf{R}$ to a closed subset of $S^{2}$. Let $\nu$ be a continuous probability measure on $\mathbf{R}$ and

$$
\mu=\sum_{n=1}^{\infty} \frac{1}{2^{n}}\left(\varphi_{n}\right)_{*}(\nu) .
$$

Then $\mu$ is a perfect (regular) Borel measure on $S^{2}$, hence $\tau$-additive; its support is $S^{2}$ which is not Lindelöf. Therefore Theorem 4.7 is not valid for weakly $\theta$-refinable spaces.

4.9. Corollary. Let $(S, Q, \mu)$ be a perfect measure space, $X$ a regular hereditary weakly metacompact space with nonmeasurable cardinal and $f$ a $\mu$-measurable function from $S$ onto $X$. Then there is a closed Lindelöf subset $Y$ of $X$ such that $\mu(S)=\mu\left(f^{-1}(Y)\right)$.

Proof. The measure $\nu=f_{*}(\mu)$ is perfect, hence, by Corollary 4.6, regular. From Theorem 4.7 it follows that there is a closed Lindelöf $Y \subset X$ such that $\nu(Y)=$ $\nu(X)$, that is, $\mu\left(f^{-1}(Y)\right)=\mu(X)$.

4.10. Definition. A topological space $X$ is developable if it has a sequence of open covers $\mathscr{U}_{n}, n=1,2, \ldots$, such that $\cup\left\{V \in \mathcal{U}_{n}: x \in V\right\}, n=1,2, \ldots$, is a neighbourhood base of each point $x \in X$.

Every metric space is developable (take $\mathscr{U}_{n}$ to be all open sets of diameter less than $1 / n)$.

Remarks. (i) If a space $X$ is developable, then every closed subset of $X$ is a $G_{\delta}$, hence every Borel measure on $X$ is regular.

(ii) Every subspace of a developable space is developable.

(iii) Every developable and Lindelöf space is second countable.

Taking account of these remarks, Theorem 4.7 and Corollary 4.9 take the following form. 
4.11. Theorem. Let $X$ be a weakly metacompact and developable $T_{1}$-space and $\mu$ a perfect Borel measure on $X$. Then the following are equivalent:

(i) There is a closed second countable subset $Y$ of $X$ such that $\mu(Y)=\mu(X)$;

(ii) there is a subset $R$ of $X$ such that every closed discrete subset of $R$ has nonmeasurable cardinal and $\mu^{*}(R)=\mu(X)$.

4.12. Corollary. Let $(S, \mathcal{Q}, \mu)$ be a perfect measure space, $X$ a weakly metacompact and developable space and $f: S \rightarrow X$ a $\mu$-measurable function such that $|f(S)|$ is nonmeasurable. Then there is a closed second countable subset $Y$ of $X$ such that $\mu(S)=\mu\left(f^{-1}(Y)\right)$.

In [21] Prikry proves Corollary 4.12 when $\mu$ is the Lebesgue measure on $[0,1]$ extending Theorem 3.6 of Solovay.

If $X$ in Theorem 4.11 is also regular, then $Y$ is metrizable and by Proposition 3.1, $\mu$ is tight. Consequently we have the following theorem promised at the beginning of this section.

4.13. TheOREM. Theorem 3.2 and its Corollaries 3.3-3.5 remain valid when $X$ is regular, weakly metacompact and developable space.

The following corollary which is known for metric spaces (cf. [1, Theorem 6.2]) follows immediately from the above.

4.14. COROLlaRY. Let $X$ be a regular, weakly metacompact and developable space. Then the following are equivalent.

(i) $|X|$ is nonmeasurable;

(ii) every closed discrete subset of $X$ has nonmeasurable cardinal.

Proof. By Theorems 4.11 and 4.13 any of these statements is true if and only if every perfect Borel measure on $X$ is tight.

5. Perfect Baire measures. In this section all topological spaces will be assumed to be completely regular. A space $X$ is said to be measure-compact (resp. strongly measure-compact) if $M_{\sigma}(X)=M_{\tau}(X)$ (resp. $\left.M_{\sigma}(X)=M_{t}(X)\right)$. These spaces have been studied mostly by Moran ([17], [18] and [19]). It is well known that every separable complete metric space is strongly measure-compact and that every Lindelöf space is measure-compact (cf. [5, p. 40] and [28, p. 175]). Here we examine spaces for which $M_{p} \subset M_{\tau}$ and $M_{p}=M_{t}$ which have analogous properties.

The realcompactification of a space $X$ is denoted by $v X$; of course $X$ is realcompact if $X=v X$ (see [1]). In the proposition that follows it is shown that in order that $M_{p}(X) \subset M_{\tau}(X)$ a necessary condition is that $X$ be realcompact, but this condition is not always sufficient (Example 5.2). For paracompact spaces realcompactness is also a sufficient condition (Corollary 5.11), while for metric spaces it yields $M_{p}=M_{t}$ (Corollary 5.12).

\subsection{Proposition. If $M_{p}(X) \subset M_{\tau}(X)$, then $X$ is realcompact.}

Proof. The 2-valued measures on $X$ which are $\sigma$-additive and $\tau$-additive are precisely the points of $v X$ and $X$ respectively. On the other hand, every 2-valued 
measure is perfect. Therefore, if $M_{p}(X) \subset M_{\tau}(X)$ then $v X=X$, so $X$ is realcompact.

5.2. ExAmple. The Sorgenfrey plane $S^{2}$ is a realcompact space because $S$ is completely regular and Lindelöf, hence realcompact (cf. [1, Corollary 2.4]). In [17] Moran shows that $S^{2}$ is not measure-compact. Using an argument of Moran [17, p. 637] we can show that every Baire set of $S^{2}$ is a Baire set in $\mathbf{R}^{2}$. Since the topology of $S^{2}$ is finer than the usual topology of $\mathbf{R}^{2}$, we have $\mathscr{B}\left(S^{2}\right)=\mathscr{B}\left(\mathbf{R}^{2}\right)$. Therefore, $M_{\sigma}\left(S^{2}\right)=M_{\sigma}\left(\mathbf{R}^{2}\right)$ and $M_{p}\left(S^{2}\right)=M_{p}\left(\mathbf{R}^{2}\right)$. But $M_{\sigma}\left(\mathbf{R}^{2}\right)=M_{p}\left(\mathbf{R}^{2}\right)$, therefore $M_{\sigma}\left(S^{2}\right)$ $=M_{p}\left(S^{2}\right)$. Since $S^{2}$ is not measure-compact, we have $M_{p}\left(S^{2}\right) \nsubseteq M_{\tau}\left(S^{2}\right)$. Thus, the converse of Proposition 5.1 is not true.

A concrete perfect measure on $S^{2}$ which is not $\tau$-additive can be described as follows. The function $f:[0,1] \rightarrow S^{2}$ with $f(x)=(x,-x)$ is Baire measurable (since $\left.\Re\left(S^{2}\right)=\mathscr{B}\left(\mathbf{R}^{2}\right)\right)$. Let $\lambda$ be the Lebesgue measure on $[0,1]$ and $\nu=f_{*}(\lambda)$. Then $\nu$ is a perfect measure on $S^{2}$ which is supported by the discrete zero set $\{(x, y)$ : $x+y=0\}$ of $S^{2}$. Therefore the support of $\nu$ is empty, so $\nu$ is not $\tau$-additive.

Another example of a realcompact space for which $M_{p} \nsubseteq M_{\tau}$ is given in the comments following 5.6. The class of spaces for which $M_{p} \subset M_{\tau}\left(M_{p}=M_{t}\right)$ have analogous permanence properties with the class of measure-compact (strongly measure-compact) spaces which appear in [18] and [9]:

5.3. Theorem. (i) If $M_{p}(X) \subset M_{\tau}(X)$ (or $M_{p}(X)=M_{t}(X)$ ), then every Baire set and every closed set of $X$ has the same property.

(ii) The countable product of spaces for which $M_{p}=M_{t}$ has the same property.

(iii) The product of two spaces for which $M_{p} \subset M_{\tau}$ need not have the same property.

(iv) If $M_{p}(X) \subset M_{\tau}(X)$ and $M_{p}(Y)=M_{t}(Y)$, then $M_{p}(X \times Y) \subset M_{\tau}(X \times Y)$.

An example for (iii) is the space $S^{2}$. In fact, $M_{\sigma}(S)=M_{\tau}(S)$ since $S$ is Lindelöf. Therefore $M_{p}(S) \subset M_{\tau}(S)$, but $M_{p}\left(S^{2}\right) \nsubseteq M_{\tau}\left(S^{2}\right)$ (Example 5.2).

We now come to infinite products and use the following known result (cf. Ross and Stone [22]).

5.4. TheOrem. Let $\left\{X_{i}\right\}_{i \in I}$ be a family of separable spaces and $X=\Pi_{i \in I} X_{i}$. Then every continuous function $f: X \rightarrow \mathbf{R}$ is determined by countably many coordinates, i.e. there is a countable $J \subset I$ such that $x=\left(x_{i}\right)_{i \in I} \in X, y=\left(y_{i}\right)_{i \in I} \in X$ and $x_{i}=y_{i}$ for $i \in J \Rightarrow f(x)=f(y)$.

5.5. THEOREM. Let $\left\{X_{i}\right\}_{i \in I}$ be a family of nonempty separable spaces and $X=$ $\Pi_{i \in I} X_{i}$. Then $M_{\sigma}(X)=M_{p}(X)$ if and only if $M_{\sigma}\left(\Pi_{i \in J} X_{i}\right)=M_{p}\left(\Pi_{i \in J} X_{i}\right)$ for every countable $J \subset I$.

Proof. First we prove that Theorem 5.4 holds for a Baire measurable function $f$ : $X \rightarrow \mathbf{R}$. The family of subsets of $X$ with characteristic function determined by countably many coordinates is a $\sigma$-algebra of subsets of $X$. By Theorem 5.4 this $\sigma$-algebra contains all zero sets of $X$. Therefore the characteristic function of every Baire set, hence every simple Baire measurable function, is determined by countably many coordinates. The conclusion follows from the fact that every Baire measurable function is the pointwise limit of a sequence of simple functions. 
Now assume that $M_{\sigma}\left(\prod_{i \in J} X_{i}\right)=M_{p}\left(\Pi_{i \in J} X_{i}\right)$ for every countable $J \subset I$. Let $\mu \in M_{\sigma}^{+}(X)$ and $f: X \rightarrow \mathbf{R}$ be a Baire measurable function. By the above there is a countable $J \subset I$ and a Baire measurable function $g: \Pi_{i \in J} X_{i} \rightarrow \mathbf{R}$ such that $f=g \circ \Pi_{J}$, where $\Pi_{J}: X \rightarrow \Pi_{i \in J} X_{i}$ is the canonical function. By our assumption $\left(\Pi_{J}\right)_{*}(\mu) \in M_{p}\left(\Pi_{i \in J} X_{i}\right)$. So there is $B \in \mathscr{B}(\mathbf{R})$ such that $B \subset g\left(\Pi_{i \in J} X_{i}\right)=f(X)$ and $\left(\Pi_{J}\right)_{*}(\mu)\left(g^{-1}(B)\right)=\left(\Pi_{J}\right)_{*}(\mu)\left(\Pi_{i \in J} X_{i}\right)$, i.e. $\mu\left(f^{-1}(B)\right)=\mu(X)$. Therefore $\mu$ is perfect and we have shown that $M_{\sigma}(X)=M_{p}(X)$.

Conversely, assume that $M_{\sigma}(X)=M_{p}(X)$ and let $\mu \in M_{\sigma}\left(\Pi_{i \in J} X_{i}\right)$ for some $J \subset I$ countable. There is a homeomorphism $h$ of $\prod_{i \in J} X_{i}$ onto a subspace of $X$ such that $\Pi_{J} \circ h$ is the identity function of $\Pi_{i \in J} X_{i}$. We identify $\Pi_{i \in J} X_{i}$ with this subspace. If $B$ is a Baire set of $\prod_{i \in J} X_{i}$, then $B=\left(\prod_{i \in J} X_{i}\right) \cap \Pi_{J}^{-1}(B)$, where $\Pi_{J}^{-1}(B)$ is a Baire set of $X$. Therefore we have

$$
\mathscr{B}\left(\prod_{i \in J} X_{i}\right)=\left(\prod_{i \in J} X_{i}\right) \cap \mathscr{B}(X) .
$$

Let $\bar{\mu}$ be the image of $\mu$ to $X$. By our assumption $\bar{\mu} \in M_{p}(X)$. It follows from (*) and Proposition 1.1 that $\mu=\left.\bar{\mu}\right|_{\Pi_{i \in J} X_{i}} \in M_{p}\left(\prod_{i \in J} X_{i}\right)$.

Theorem 5.5 can be generalized since the only property of the product space $X$ which is actually used is that every real-valued continuous function on $X$ is determined by countably many coordinates, which is valid under weaker hypotheses (see [20]). We restrict ourselves to products of $\mathbf{R}$ and $\mathbf{N}$ (with the discrete topology) and have immediately the following.

5.6. Corollary. $M_{\sigma}\left(\mathbf{R}^{\alpha}\right)=M_{p}\left(\mathbf{R}^{\alpha}\right)$ and $M_{\sigma}\left(\mathbf{N}^{\alpha}\right)=M_{p}\left(\mathbf{N}^{\alpha}\right)$ for every cardinal number $\alpha$.

Kemperman and Maharam [8] prove that $\mathbf{N}^{c}$ is not measure-compact. Since $\mathbf{N}^{c}$ is homeomorphic to a closed subset of $\mathbf{R}^{c}$, the same is true for $\mathbf{R}^{c}$. A different proof for $\mathbf{R}^{c}$ is given by Moran [17]. It follows from Corollary 5.6 that $M_{p}\left(\mathbf{R}^{c}\right) \notin M_{\tau}\left(\mathbf{R}^{c}\right)$ and $M_{p}\left(\mathbf{N}^{c}\right) \nsubseteq M_{\tau}\left(\mathbf{R}^{c}\right)$, although $\mathbf{R}^{c}$ and $\mathbf{N}^{c}$ are realcompact spaces.

The following theorem appears in [3, Theorem 8.8] under the hypothesis that $X$ is measure-compact. Its proof, using results for $M_{p}$ instead of $M_{\sigma}$ (i.e. Proposition 5.1, Theorem 5.3(i) and $\left.M_{p}\left(\mathbf{N}^{c}\right) \not M_{\tau}\left(\mathbf{N}^{c}\right)\right)$ is like this result.

5.7. TheOREM. Let $\left\{X_{i}\right\}_{i \in I}$ be a family of nonempty topological spaces and $X=\prod_{i \in I} X_{i}$. If $M_{p}(X) \subset M_{\tau}(X)$, then $\mid\left\{i \in I: X_{i}\right.$ is not compact $\} \mid<c$.

The analogues of Theorems 4.3 and 4.7 are not correct for Baire measures. Indeed, Moran in [19, Example 7.1] gives an example of a metacompact space $X$ which is not realcompact and $|X|=\aleph_{1}$. Therefore every Borel measure on $X$ is $\tau$-additive (cf. Theorem 4.5), but $M_{p}(X) \nsubseteq M_{\tau}(X)$ (Proposition 5.1). Also, $S^{2}$ is an example of a weakly $\theta$-refinable realcompact space for which every perfect Borel measure is $\tau$-additive and $M_{p}\left(S^{2}\right) \nsubseteq M_{\tau}\left(S^{2}\right)$ (5.2 and 4.8). However, for paracompact spaces the situation is different. We will need the following two results. The first is the analogue of Lemma 4.2(ii) on Borel measures and the second is due to Marik [14]. We recall that a space $X$ is countably paracompact if every countable open cover of $X$ has a locally finite refinement. It is also known that a space $X$ is 
paracompact if and only if every open cover of $X$ has a $\sigma$-discrete refinement and that every paracompact space is normal.

5.8. Lemma. If $\mu \in M_{\sigma}^{+}(X) \backslash M_{\tau}^{+}(X)$, then there is a zero set $Z \subset X$ such that $\mu_{Z}$ is a nonzero measure with empty support.

5.9. Proposition. Let $X$ be a normal countably paracompact space. Then every $\mu \in M_{\sigma}^{+}(X)$ has a unique regular Borel extension $\tilde{\mu}$ specified by

$$
\tilde{\mu}(G)=\sup \{\mu(Z): Z \text { zero set, } Z \subset G\}
$$

for all open $G \subset X$.

5.10. TheOrem. Let $X$ be a paracompact space and $\mu \in M_{p}(X)$. Then, $\mu \in M_{\tau}(X)$ if and only if there is a closed $R \subset X$ such that every closed discrete subset of $R$ has nonmeasurable cardinal and $|\mu|^{*}(R)=|\mu|(X)$.

Proof. The condition of the theorem in order that $\mu$ be $\tau$-additive is necessary, since it is well known that the support $C$ of a $\tau$-additive measure $\mu$ on a paracompact space $X$ is Lindelöf and $|\mu|^{*}(C)=|\mu|(X)$ (cf. [28, Part I, Theorem 27]).

In order to prove that the condition is sufficient we may assume, without loss of generality, that $\mu$ is positive. Suppose (for the purpose of contradiction) that $\mu$ is not $\tau$-additive and there is a closed $R \subset X$ such that every closed discrete subset of $R$ has nonmeasurable cardinal and $\mu^{*}(R)=\mu(X)$. By Lemma 5.8, there is a zero set $Z$ such that $\nu=\mu_{Z} \neq 0$ has empty support. For each $x \in X$ let $V_{x}$ be a cozero set such that $x \in V_{x}$ and $\nu\left(V_{x}\right)=0$. Since $X$ is paracompact we can find a $\sigma$-discrete refinement $C$ of the open cover $\left(V_{x}\right)_{x \in X}$ of $X$. Let $\mathcal{C}=\cup_{n=1}^{\infty} C_{n}$, where each $\bigodot_{n}$ is discrete.

By Proposition 5.9, $\nu$ has a regular Borel extension $\tilde{\nu}$ such that $\tilde{\nu}(G)=\nu_{*}(G)$ for every open set $G \subset X$. We have $\tilde{\nu}(R)=\nu^{*}(R)=\nu(X)=\tilde{\nu}(X)>0$.

We fix an $n$ such that $\tilde{\nu}\left(\cup \mathcal{C}_{n}\right)>0$ and let $\tilde{C}_{n}=\left\{G \in \mathcal{C}_{n}: G \cap R \neq \varnothing\right\}$. For every $G \in \tilde{\mathcal{C}}_{n}$ we choose a point $x_{G} \in G \cap R$ and let $S=\left\{x_{G}: G \in \mathcal{C}_{n}\right\}$. Then $S$ is closed and discrete (since $\tilde{C}_{n}$ is a discrete family of open sets). Therefore, $|S|=\left|\tilde{e}_{n}\right|$ is nonmeasurable and $\tilde{\nu}\left(\cup \tilde{e}_{n}\right)=\tilde{\nu}\left(\cup \mathcal{C}_{n}\right)>0$. Let $B$ be a zero set such that $B \subset \cup \tilde{\bigodot}_{n}$ and $\tilde{\nu}(B)>0$. Since $X$ is normal, we have $\mathscr{B}(B)=B \cap \mathscr{B}(X)$, so $\left.\nu\right|_{B}$ is defined on $\mathscr{B}(B)$ and it is a perfect (Proposition 1.1) nonzero measure on $B$. The family $\mathscr{B}=\left\{B \cap G: G \in \tilde{\mathcal{C}}_{n}\right\}$ is a partition of $B$ of open sets with $\nu$-measure zero. Moreover, the union of every subfamily of $\mathscr{B}$ is open-closed in $B$, so a Baire set in $B$, which contradicts Theorem 2.5 .

The equivalence (ii) $\Leftrightarrow$ (iii) in the following corollary is the Katetov's theorem [7] on paracompact spaces. The 2-valued measures which lie in $M_{p}(X)$ and $M_{\tau}(X)$ are precisely the points of $v X$ and $X$ respectively. Thus, the equivalence to (i) can be considered as an extension of Katetov's theorem.

5.11. Corollary. For every paracompact space $X$ the following are equivalent:

(i) $M_{p}(X) \subset M_{\tau}(X)$;

(ii) $X$ is realcompact;

(iii) every closed discrete subset of $X$ has nonmeasurable cardinal. 
Proof. (i) $\Rightarrow$ (ii) is Proposition 5.1. It is well known that a closed subset of a realcompact space is realcompact and that a discrete space has nonmeasurable cardinal if (and only if) it is realcompact. Therefore (ii) $\Rightarrow$ (iii). These implications are valid for every space $X$, while (iii) $\Rightarrow$ (i) follows from Theorem 5.10.

The above corollary should be compared with [28, Part I, Theorem 28] and [9, Theorem 4.5] in combination with the comments at the beginning of $\$ 4$ about Theorem 4.3.

If $X$ is a realcompact metric space then $|X|$ is nonmeasurable (cf. [1, Theorem 6.2] or Corollary 4.14). Also $\mathscr{B}(X)=\mathscr{B}_{0}(X)$. Thus, the following corollary is essentially a restatement of Corollary 3.3. It follows immediately from Corollary 5.11 and Proposition 3.1.

5.12. Corollary. A metric space $X$ is realcompact if and only if $M_{p}(X)=M_{t}(X)$.

This corollary can be extended to some nonmetric spaces. Suppose that the space $X$ has the following property: there is a Baire measurable function $f$ from $X$ onto a realcompact metric space $Y$ such that $f^{-1}(K)$ is compact for every compact $K \subset Y$. Then $M_{p}(X)=M_{t}(X)$. Indeed, let $\mu \in M_{p}^{+}(X)$ and $\varepsilon>0$. Then $f_{*}(\mu) \in$ $M_{p}(Y)=M_{t}(Y)($ Corollary 5.12), so there is a compact $K \subset Y$ such that

$$
f_{*}(\mu)(Y \backslash K)<\varepsilon,
$$

that is, $\mu\left(X \backslash f^{-1}(K)\right)<\varepsilon$ where $f^{-1}(K)$ is a compact Baire set of $X$. Therefore $\mu \in M_{t}(X)$. From this remark it follows that

(a) Corollary 5.12 remains valid when $X$ is a paracompact $M$-space, that is, when there is a perfect function from $X$ onto a metric space $Y$;

(b) if $X$ is a continuous image of a separable metric space, then $M_{p}(X)=M_{t}(X)$.

To see (a) it is enough to observe that, by Katetov's theorem, the metric space $Y$ is realcompact whenever $X$ is realcompact. For (b), we have that there is a Baire measurable 1-1 function $g$ from $X$ onto a separable metric space $Y$ such that $g^{-1}$ is continuous. Indeed, we have that $X$ is hereditary Lindelöf, so every open set is a cozero set. Therefore $\mathscr{B}(X)=\mathscr{B}_{0}(X)$. Also, from [15, Proposition 10.2] it follows that there is a countable family $\bigodot$ of subsets of $X$ such that every open subset of $X$ is a union of some members of $\mathcal{C}$. By the regularity of $X$ we may suppose that the

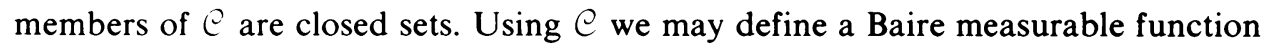
$g: X \rightarrow\{0,1\}^{\mathbf{N}}$ as in the proof of Theorem 2.1. Then $g$ from $X$ onto the separable metric space $Y=g(X)$ is Baire measurable, 1-1 and open; hence $g^{-1}$ is continuous.

Note ADDED IN PROOF. Since submitting this paper the author has learned that some of the results of $\S 3$ and related results have been obtained by D. Fremlin, Measurable functions and almost continuous functions, preprint, A. Goldman and M. Talagrand, Propriétés de Radon-Nikodym pour les cônes positifs de mesures, Math. Scand. 44 (1979), 345-356, and J. Pachl, Two classes of measures, Colloq. Math. 42 (1979), 331-340.

\section{REFERENCES}

1. W. W. Comfort and S. Negrepontis, Continuous pseudometrics, Marcel Dekker, New York, 1975.

2. N. Dinculeanu, Vector measures, Pergamon Press, New York, 1967. 
3. R. J. Gardner, The regularity of Borel measures and Borel measure-compactness, Proc. London Math. Soc. (3) 30 (1975), 95-113.

4. R. Haydon, On compactness in spaces of measures and measurecompact spaces, Proc. London Math. Soc. (3) 29 (1974), 1-16.

5. P. R. Halmos, Measure theory, Van Nostrand, Princeton, N. J., 1960.

6. T. Jech, Set theory, Academic Press, New York, 1978.

7. M. Katetov, Measures in fully normal spaces, Fund. Math. 38 (1951), 73-84.

8. J. H. B. Kemperman and D. Maharam, $R^{c}$ is not almost Lindelöf, Proc. Amer. Math. Soc. 24 (1970), 772-773.

9. R. B. Kirk, Measures in topological spaces and B-compactness, Indag. Math. 31 (1969), 172-183.

10. __ Locally compact, B-compact spaces, Indag. Math. 31 (1969), 333-344.

11. J. D. Knowles, Measures on topological spaces, Proc. London Math. Soc. (3) 17 (1967), 139-156.

12. D. J. Lutzer, Another property of the Sorgenfrey line, Compositio Math. 24 (1972), 359-363.

13. E. Marczewski, On compact measures, Fund. Math. 40 (1953), 113-124.

14. J. Marik, The Baire and Borel measure, Czechoslovak Math. J. 7(82) (1957), 248-253.

15. E. Michael, $\mathbb{N}_{0}$-spaces, J. Math. Mech. 15 (1966), 983-1002.

16. D. Montgomery, Non-separable metric spaces, Fund. Math. 25 (1935), 527-533.

17. W. Moran, The additivity of measures on completely regular spaces, J. London Math. Soc. 43 (1968), 633-639.

18. Measures and mappings on topological spaces, Proc. London Math. Soc. (3) 19 (1969), 493-508.

19. __ Measures on metacompact spaces, Proc. London Math. Soc. (3) 20 (1970), 507-524.

20. N. Noble and M. Ulmer, Factoring functions on cartesian products, Trans. Amer. Math. Soc. 163 (1972), 329-339.

21. K. Prikry, On images of the Lebesgue measure. I, II, III, manuscripts, 1977.

22. K. A. Ross and A. H. Stone, Products of separable spaces, Amer. Math. Monthly 71 (1964), 398-403.

23. C. Ryll-Nardzewski, On quasi-compact measures, Fund. Math. 40 (1953), 125-130.

24. V. V. Sazonov, On perfect measures, Amer. Math. Soc. Transl. (2) 48 (1965), 229-254.

25. R. M. Solovay, A model of set theory in which every set of reals is Lebesgue measurable, Ann. of Math. (2) 92 (1970), 1-56.

26. _ Real-valued measurable cardinals, Axiomatic Set Theory, Proc. Sympos. Pure Math., vol. 13, Part I, Amer. Math. Soc., Providence, R. I., 1971, pp. 397-428.

27. S. Ulam, Zur Masstheorie in der allgemeinen Mengenlehre, Fund. Math. 16 (1930), 140-150.

28. V. S. Varadarajan, Measures on topological spaces, Amer. Math. Soc. Transl. (2) 48 (1965), 161-228.

29. R. E. Zink, On the structure of measure spaces, Acta Math. 107 (1962), 53-71.

Department of Mathematics, Athens University, Athens 621, Greece

Current address: School of Mathematics, University of Minnesota, Minneapolis, Minnesota 55455 